\title{
Design Research of Silica Soft Mold on SMARTM
}

\author{
Zhanjun Liu \\ Faculty of Aerospace Engineering \\ Shenyang Aerospace University \\ Shenyang, China \\ liuzhanjundef@163.com
}

\begin{abstract}
The SMARTM compound materials formation technique characteristic is summarized, taking some compound materials connection skirt as the example, it designs SMARTM with the mold structural style, it has inferred the soft mold outer diameter and thickness design and the dimension control equation. The research analyzed the silica gel soft mold being heated inflation compression process sealed up in the cavity, it discovered the silica gel soft mold the inflation traveling schedule, the soft mold outer diameter only connected the skirt with the compound materials the size, the layer fabric density.
\end{abstract}

Keywords-SMARTM; soft silica; size design; formation craft; bulging forming

\section{INTRODUCTION}

RTM process is a kind of rigid closed die manufacturing composite materials technology, its basic principle is that in the mold cavity, reinforced material is placed in advance, after the mould clamping, certain temperature and pressure are in the static mixer mixing system of resin injection mould, which is dipping reinforcing fabric and curing, the composites molding process of the final releases products. As the development of low viscosity for the aircraft industry, application progress is thermosetting resin, made by RTM laminated, the plates can be close to the hot pressing process of presoak laminate material mechanical properties [1]. Advanced composite missile parts is make progress, which mainly depends on the improvement parts manufacturability, RTM process is the best choice of achieving this goal. However, RTM process development time is shorter, inevitably there are difficulties to solve the problem. RTM technology is at home and abroad, the difficulties and problems of the common is mainly manifested in three aspects, resin impregnation of fiber is not ideal, the products of the fiber volume fraction is not high, generally no more than $50 \%$, within the area, complex mold cavity is molding resin flow in the process of imbalance, it is difficult to forecast and control. Domestic is in addition to these problems, there is difficult to meet the RTM resin special resin. In recent years, aiming at the existing problem of RTM and limitations, both at home and abroad have carried out a great deal of research, which makes the RTM technology more mature, form a complete material, process and theory system. Mainly reflected is in the compaction reinforcement and supplemented by high vacuum measures, which further improves the fiber volume fraction of the product[2]. The multi-dimensional weaving technology is preforming technology.

\section{The Soft Mode OF Assisted RTM}

SMARTM process is the RTM process that soft mode is expansion molding process together, which uses two kinds of process technology advantage to integral form of high performance internal tubular products containing complex structure[3]. TEM technique is based on the characteristics of products, to design rigid female die and flexible core modules. Inside the cavity, die cavity size is for the appearance of composite materials products size, the shape of the mandrel size is similar to inner cavity shape composite material products, but size should be less than the real size of products, to reduce the size of the called process clearance. In composites molding, presoak material is on the core of the mould, and then it closed cavity die pressurized solidification. The general process principle of TEM as follows, free expansion section, mandrel expansion drive presoak material filling process clearance, in the initial pressure section, core mold is expanded extrusion presoak material to meet the design size of material products, in constant pressure constant temperature period, mandrel expansion volume and pressure are constant, in the cool step-down section, as the temperature drops, core mold shrinkage, stress removal.

In SMARTM method, forming on the surface of the composite cylinder body of male mold design is done as a combination of rigid and soft module of support structure. Soft mode is suit in the rigid support surface, by rigid support in the electric heating device for heating body, it make the soft mode expansion, to form cylinder body surface, and it use a rigid structure, cavity die in soft mode when inflation is still high stiffness[4], which ensure the accuracy of the product appearance size. Reinforced material layer adopts dry yarn or fiber reinforced preforming body instead of presoak material, it is layer on the soft mode, clamping, sealing, resin into the cavity by RTM injection machine, in the condition of soft mode of heat expansion, curing is to be composites.

\section{THE SOFT MOUld STRUCTURE DESIGN}

Except combination in the male mold of SMARTM technology in die have soft mode, other forms of structure and VARTM with mold, in a certain type of composite connection skirt as an example, the design of SMARTM is in the form of a mould structure, it is as shown in Fig .1.

This form of soft mode expansion and extruded process is as follows, in room temperature, the reinforced material 
is silicone rubber soft mode with the nested in rigid die cavity, to seal after injection of resin, and mould makes silicone rubber soft mould heat core expansion[5], before reinforced fiber resin system produces is about $2 \mathrm{Mpa}$ pressure, extrusion is composite component, crowded to go part of the resin, which improves the fiber volume fraction and mechanical properties.

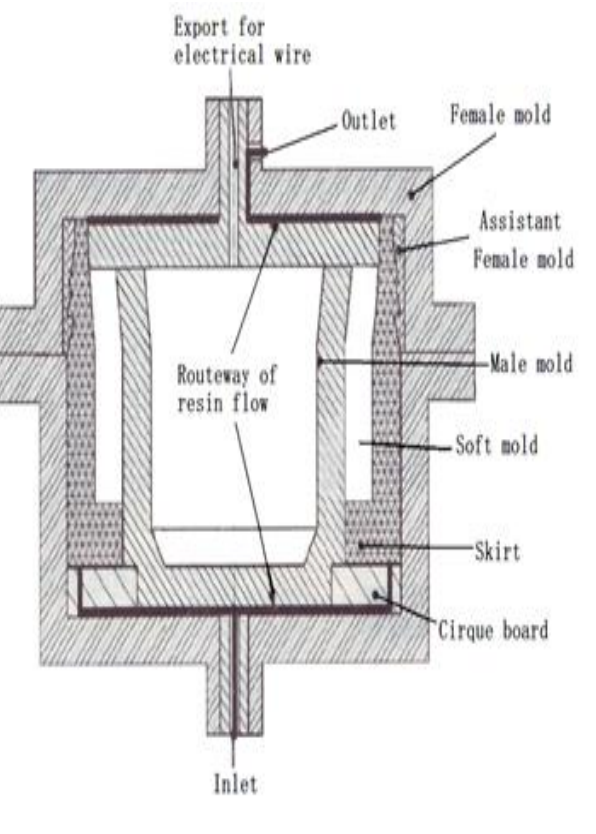

Figure 1. The mold structural style for SMARTM

\section{The Soft Mode Design AND Size OF CONTROLLING EQUATION DIAMETER}

Compared various mold material coefficient of thermal expansion, the thermal expansion coefficient of silicone rubber is much greater than the ductile cast iron, that is to say, in the silicone rubber SMARTM process, thermal expansion deformation can be ignored in rigid female die and male die core nodular cast iron material. Based on the characteristics of mold structure and silicone rubber thermal expansion, technology can get fiber reinforced the original thickness and module of preforming process clearance expansion, expansion is the soft mode before the diameter in front of the delta, soft mold is wall thickness and diameter expansion after the soft mode[6], the molding of composite connection is skirt diameter, after forming the thickness, the fiber reinforced composite material connection skirt products, after preforming body is compaction thickness silicone rubber soft mode expansion stroke. Fig .2 is the size relations between molds and product section plane.

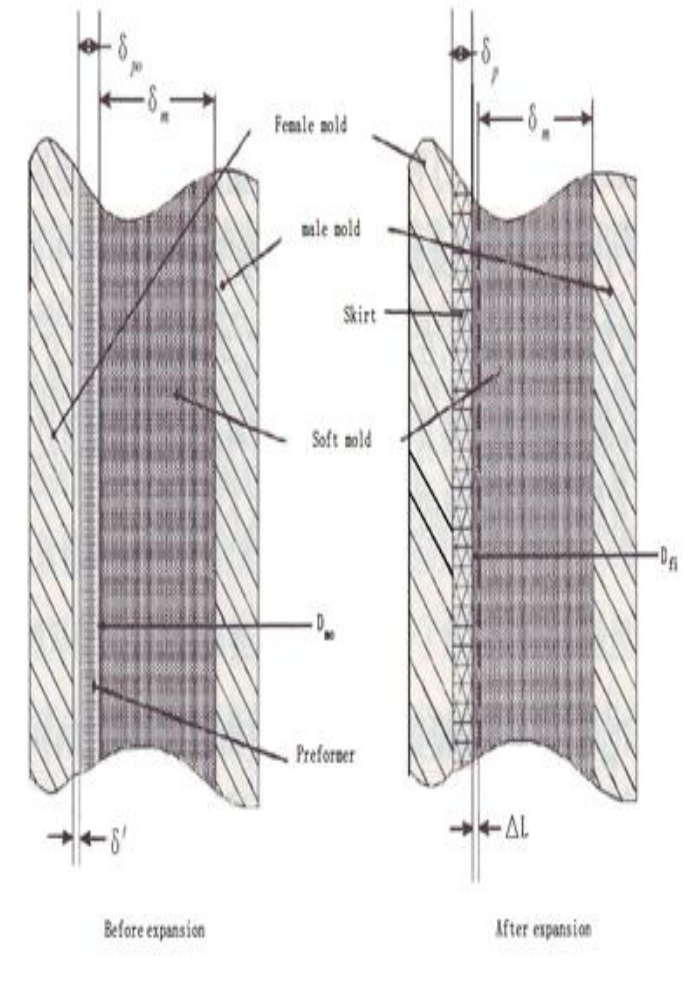

Figure 2. Size relations between molds and product section plane

\section{The Composite Material of Plane Parts FORMING TECHNOLOGY MAINLY INCLUDES}

\section{A. Molding Technologies of Resin Transfer}

The main principle of RTM process is laying reinforced material form in the cavity, in accordance with the requirements of performance and structure, injection equipment will be used by special resin system of closed cavity, mold has fastening[7], injection mould with peripheral seal and exhaust system, which ensures that the resin flow smooth out all the gas in the cavity side by side and infiltrating fiber thoroughly, it also has heating system, which is used as heating composite components of.curing forming. It is a kind method of not using presoak expected, also don't use the autoclave shaping. Therefore, which has the advantages of high efficiency, investment, green, it is a new generation manufacturing technology of future aircraft body with development potential.

\section{B. The Molding Technology of Resin Transfer Is A Kind of Low Cost Composite Materials}

The original of manufacturing methods is mainly used for aircraft bearing structures, such as doors and check flap. It carried out the high strength of the main bearing components of RTM low cost manufacturing technology research[8]. The small and medium-sized composite RTM parts manufacturing has obtained the widespread application, and large RTM parts is successfully on the tail of the JSF application. This method has the advantage of good performance for environmental protection, the formation of the laminated plates and double are of good quality and applied in the air can not only reduce labor itself, and due to forming large pieces, to reduce the 
assembly work. But resin injection into the mold cavity through pressure formed parts, there is a larger pore content and fiber content is low, distribution of resin in the fiber is uneven, defects is such as inadequate resin impregnation of the fibers, so the technology has potential for improvement.

Because the technology also exists above shortcomings, so future development is to reduce tooling cost, improve the structure performance and reduce scrap. Therefore, based on the technology[9], it has developed the vacuum assisted resin injection forming (VARI) technology, which auxiliary resin is absorbed by the fabric, not only can decrease the porosity, fiber preform closer, owing to vacuum negative pressure, resin is suitable vacuum channel along the flow into the form all levels, thus fully impregnated fiber, and make the fiber/resin evenly distributed. To improve the performance, it is mainly to use RTM manufacturing high strength parts, therefore, the national developed Z fiber reinforced RTM technology, the technology can be used at the interface between fabric transverse or enhance skin discontinuous fiber reinforced. This new type of manufacturing theory is not through weaving or closing a three-dimensional structure. The technology has several key technologies in the process of application to solve: filling process simulation technology, that is heat transfer and curing reaction research, injection methods, RTM equipment.

\section{MOLDING TECHNOLOGIES OF RELATIVE POSITION IS WITH FLARING RATIO}

The influence is pipe end position, when the end of pipe is beyond $10 \mathrm{~mm}$, magnetic pressure is almost zero. When the coil is no taper, it won't make the pipe deformation. When the Lc is negative, tube is expanding into a cone. Pipe relations of relative position is with flaring ratio . Regardless of the charging energy[10], when $\mathrm{Lc} / \mathrm{R}$ is 0.5 , pipe belling is the most. When $\mathrm{Lc} / \mathrm{R}$ is less than 0.5 , the pipe belling ratio decreases with the decrease of the $\mathrm{Lc} / \mathrm{R}$, when $\mathrm{Lc} / \mathrm{R}$ is greater than 0.5 , and which decreases due to the distance between the tube and coil the magnetic pressure of acting on the pipe, thus it reducing the flaring. As a taper coil is zero, due to the distance between the tube and coil, the basic is constant is flaring.

The taper pipe forming length is equal to zero by the coil in flaring, no matter how high, forming length is under the same discharge energy, tube and pipe near the clamping frame deformation is nearly the same, and the middle part is in the shape of straight uniform flaring, such as using coil with a taper shape, regardless of the length of the forming, the pipe is clamping frame, which is the same as in other parts of the deformation.

In the electromagnetic forming process, most of the deformation is in pulse pressure disappears. Under the effect of pulse pressure, the metal is obtained great acceleration, and got a lot of kinetic energy, metal forming is mainly depended on the kinetic energy to achieve. The electromagnetic forming is with pure electromagnetic properties, its forming process is not affected by mechanical inertia of the moving parts, so the electromagnetic forming can achieve rapid processing, which can work hundreds of times per minute. And the intensity of magnetic pulse can be accurately controlled, which is easy to realize mechanization and automation[4]. The magnetic field can penetrate the nonconductive material, so to have a non-metallic coating or in forming the part with container. The electromagnetic forming is medium pressure on the workpiece with magnetic field, which is no mechanical contact. So the workpiece does not produce friction, which is no lubrication.The electromagnetic forming is a single-mode forming, which simplified the mould manufacturing, to increased the processing flexibility. The electromagnetic forming parts are with high precision, residual stress low, and the springback is small. The process of materials are best used in below $0.15 \mathrm{um}$, the high resistance materials need to be adopt the high frequency equipment, the driven piece of structure is used on the tooling. Electromagnetic forming is often used in flat workpieces and the forming of tube blank.

The section size of distributary channel mainly depends on the size of the plastic products, mould structure and processing of plastic types. In general, the increase of product size is wall thickness, because the melt is in the big cross section, small flow channel section flows in the runner when the resistance is small, therefore, large cross section flows, channel can promote the mould filling process. If shunt way is longer, the process is longer, plastic viscosity should be smaller. In addition, the products of the production of economy should also be considered, otherwise, compared with the model of product, distributary channel cross section is too big, it will affect the cooling capacity and cooling time, which is causing the waste of material and time. Due to the size of the diversion channel, the product quality and production efficiency have a significant impact, therefore, the general points and the diameter of the sprue are within $3 \sim 10 \mathrm{~mm}$. And distributary channel is length between $8 \sim 30 \mathrm{~mm}$, it can be also according to the number of cavity appropriate lengthen, but it should not be less than $8 \mathrm{~mm}$, otherwise, it is difficult to repair the mould. The section size of distributary channel is according to the variety of products used in plastic, weight, thickness and the length of the shunt way to determine. The look up table shows that the density of ABS plastic is $1.01 \sim 1.08$, the calculation of plastic volume is for $98 \mathrm{~g}$. So the quality of plastic is $102 \mathrm{~g}$. Because the wall thickness of parts is for $5 \mathrm{~mm}$, for ABS plastic, in order to make the shunt way easy machining and easy to launch, coagulation is for $6 \mathrm{~mm}$ in diameter.

Cold rolled metal industry requires reliable and accurate model formula to improve the surface finishing and friction prediction ability, thus improve the yield and quality. But with cold rolled metal, surface friction factor is very complexity, so far, its theory is also approximate model of the formula, it is necessary for the metal surface of cold rolling friction simulation, and prediction results and experiment comparison are based on the predecessors of the test of semi-empirical formula to further improve the prediction ability of surface friction, this paper takes AISI 1005 carbon steel as an example, which is carried out a research into this aspect to explore, it is of great 
significance to improve the forecast ability of metal surface friction cold-rolled industry.

Because contact area usually is larger than the bottom area, and the friction factor of contact area is higher, so the influence from contact areas will plays a decisive role. So accurate representation is very important. The current typical numerical model is used about $\mathrm{u}_{\mathrm{c}}$ and $\mathrm{u}_{\mathrm{v}}$. For the improvement of the model, it is required to determine the coefficient of friction factor, and smooth oil film thickness or the relationship between the rolling speed. The reason behind this approach is friction in contact area under some fluid composition. Montmitonnet is deduced from mill trials an appropriate variable friction coefficient, who put forward the experience of the law of friction and a half, and it is using a simple strip drawing test to launch a contact friction coefficient under smooth oil film thickness.

The experiment have good consistency with both prediction results and, which suggests that with the increase of rolling speed and lubrication parameters, friction coefficient is from high to low transition. Semiempirical model of the benefits of doing is simple to use, but it needs to be the experimental data as the input values. Double wave model, although it is difficult to implement, don't need too much empirical data input, so it has become a very valuable research tool, which is used for calibration certificates and semi-empirical model.

\section{CONCLUSIONS}

The SMARTM compound materials formation technique characteristic is summarized, taking some compound materials connection skirt as the example, it designs SMARTM with the mold structural style, it has inferred the soft mold outer diameter and thickness design and the dimension control equation. The research analyzed the silica gel soft mold being heated inflation compression process sealed up in the cavity,it discovered the silica gel soft mold the inflation traveling schedule, the soft mold outer diameter only connected the skirt with the compound materials the size, the layer fabric density.

\section{REFERENCES}

[1] Gutowski T G,Advanced composites manufacturing, John Wiley \& Sons Inc, New York, pp.416-429,1997.

[2] Rudd C D, Long A C, Kendall K N, Mangin C G E, Liquid molding technologies, Woodhead Publishing, London, pp. 203$253,1997$.

[3] White Frank M, Fluid mechanics (third edition), McGraw-Hill Inc, NJ, Highstown,pp.221 -222,1994.

[4] Middleman S, An introduction to fluid dynamics ,John Wiley and Sons Inc, New York, pp.16-17,1998.

[5] LiuZhanJun,Difficulty reliability prediction research of titanium plate stamping forming based on the fuzzy control, plastic engineering journal, Beijing, pp. 63-66,2005.

[6] Feng. Jing , The Mixture of The Multimode System Reliability Growth Model,Computer Application Research Supplement, pp.60-61, 2003.

[7] Xu. Yuxiu, Complex Mechanical Fault Diagnosis Method of Fractal and Wavelet, Mechanical Industry Publishing House,Beijing, pp.24-25,2005.

[8] Weng. chaoxi, Reliability Growth, Science Press, Beijing, pp.130131,1993 .

[9] Guofang, Reliability Data Collection And Analysis, National Defence Industry Press, p22,1995.

[10] Wen.WeiDong, Prediction model based on the fuzzy reliability aircraft engine, Beijing, pp.322-323,2003. 